\title{
Antidepressant-Like Effects of Ceftriaxone in Chronic Unpredictable Mild Stress Model in Rats: The Importance of Reuptake Time of Glutamate
}

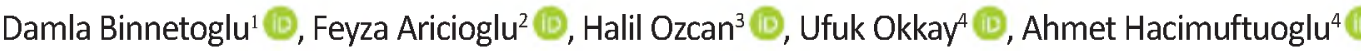 \\ ${ }^{1}$ Kafkas University, Faculty of Medicine, Department of Pharmacology \\ ${ }^{2}$ Marmara University, Faculty of Pharmacy, Department of Pharmacology and Psychopharmacology Research Unit \\ ${ }^{3}$ Ataturk University, Faculty of Medicine, Department of Psychiatry \\ ${ }^{4}$ Ataturk University, Faculty of Medicine, Department of Pharmacology \\ Correspondence Author: Damla Binnetoglu \\ E mail: damlacetin.erz@gmail.com
}

Received: $01.09 .2019 \quad$ Accepted: 19.09 .2019

\begin{abstract}
Objective: We aimed to indicate the relationship between depression and glutamate, and to reveal the effect of escitalopram, an antidepressant, which is widely used in depression treatment and reuptake parameters of glutamate, and to treat depression with ceftriaxone, one of the beta lactam antibiotics which increased the number and activity of glutamate transporters.

Methods: In CUMS, rats subjected to series of different mild stressors in an unpredictable manner for 40 days. On the day 20 rats were divided in to groups such as CUMS, CUMS+Escitalopram and CUMS+Ceftriaxone. 4 weeks. Treatments were started at 2nd week of CUMS and continued for 21 days. Anhedonia and antidepressant effect were assessed by sucrose preference (SP), locomotor activity (LA), elevated plus maze (EPM) and forced swim test (FST) at the end of the experiment respectively. At the end of the experiment, behavioral tests were made, and glutamate reuptake time in CA3 (cornuammonis 3) brain region which are related with depression were measured by means of in vivo voltammetry technique.

Results: Ceftriaxone treatment had an antidepressant-like effect. Escitalopram and ceftriaxone increased SP and locomotor activity, reduced immobility FST, forced swim and time spent in closed arms in EPM compared to CUMS group. In this in-vivo voltametric study, it was also observed that there was a significant decrease in glutamate reuptake time in depression.

Conclusion: Escitalopram and ceftriaxone demonstrated antidepressant-like effects by reversing behavioral changes in CUMS model. Escitalopram treatment in CA3 region corrected the decrease in glutamate reuptake time which is consistent with the hypothesis that enhanced uptake of glutamate might have antidepressant-like effects.

Keywords: Beta lactam antibiotics, ceftriaxone, depression, voltammetry, glutamate
\end{abstract}

\section{INTRODUCTION}

Major depressive disorder (MDD) is a common and chronic, recurring mental illness(1). MDD is affecting nearly 340 million people worldwide and is considered to be an underlying cause in $35-40 \%$ of suicides (2). MDD is characterized by depressed mood; anhedonia, appetite changes leading to weight gain or loss, sleep dysregulation, psychomotor changes, loss of energy, feelings of worthlessness and excessive guilt, diminished concentration and recurrent thoughts of death (3).At present, the pathophysiology of many psychiatric disorders is still not fully understood. Current treatment approaches that have been used in psychiatric disorders for many years and usually have a single mechanism-based effect are inadequate to control disease symptoms; modulation of monoamines for depression. Patients who do not respond to the current treatment options in the majority of depression which is affecting $10 \%$ of the population and resistance cases constitute about $1 / 3$ of them (4).

The mechanisms driving pathophysiology are complex and remain largely unknown, advances in the understanding of neurotransmitter activities are providing significant clues to their etiology.Moreover, a considerable interest in nonmonoaminergic approaches to the treatment of depression and glutamate modifying therapies have become emerging targets which could be more effective and speed up the recovery of patients through fast acting compounds either as monotherapy or add-on treatment.Over the past decade, accumulating evidence has reported a positive relationship between depression and glutamate, which is the most abundant excitatory neurotransmitter in the mammalian central nervous system. Recent studies in rodent models have demonstrated that excitatory amino acid transporters (EAAT) plays a critical role in the regulation of glutamate release however, glutamate cycling rates.

Mental or physical disorders are also known to be triggered by brain anoxia or ischemia which might be due to robust release of glutamate causing the death of neurons. Although the mechanisms underlying this comorbidity are unclear, emerging evidence suggests that maladaptation of the glutamate transporter. Increased levels of glutamate are associated with a number of neurological disorders such as epilepsy, stroke, Alzheimer's disease, Huntington's disease, amyotrophic lateral sclerosis and dysfunction of glutamate 
transporters has been considered as the starting point of the cascade leading to several brain injuries $(5,6)$.

Chronic unpredictable mild stress (CUMS) is considered to be one of the most extensively validated animal model of depression that meets good face, construct and predictive validity criteria (7-9). As an environmental stress induced depression model, CUMS paradigm mimics many aspects of depression, especially it is well known for producing anhedonia-like behavior in rodents which can be easily assessed by sucrose preference or sucrose consumption tests (10). Furthermore, CUMS-induced depressive like behaviors only respond to chronic but not acute antidepressant treatments, which makes it one of the more realistic depression models (11-13). Besides, the involvement of neuroinflammation and immune mechanisms in depression has been well studied in CUMS model. In this respect, CUMS-induced inflammation and lately the inflammasome activation has been widely reported by numerous studies $(14,15)$.

Recently limited number of studies indicated that ceftriaxone, a $\beta$-lactam antibiotic, treatment may enhance the expression of EAATs and prevent neurons in related pathologies. CEF has neuroprotective effects in both in vitro and in vivo models by inhibiting neuronal cell death (16). Based on the above mentioned hypothesis of depression, current study designed to investigate i) possible effect of ceftriaxone in a well-known validated animal model of depression and ii) possible role of ceftriaxone on glutamatergic transmission through EAATs in comparison with selective serotonine reuptake inhibitor escitalopram.

\section{MATERIAL AND METHODS}

\subsection{Animals and drugs}

Male adult Sprague-Dawley rats (290-320 g) were used in the present study which were supplied from Ataturk University Medical Application and Research Center (Erzurum, Turkey). Rats were housed in groups ( $n=4-5$ per each cage) under standard laboratory conditions (12 h light- 12 h dark cycle; room temperature $21 \pm 2^{\circ} \mathrm{C}$ ). Food and water were provided ad libitum except the duration of CUMS. The study was approved by the Animal Ethics and Care Committee of Ataturk University (AUDHADYEK 42190979-01-02/2236). Rats were divided into 3 experimental groups: Control (non-stressed, saline-treated, $0.1 \mathrm{ml} / 100 \mathrm{~g} / \mathrm{rat}$ for 3 weeks), CUMS (salinetreated, $0.1 \mathrm{ml} / 100 \mathrm{~g} / \mathrm{rat}$ for 3 weeks), CUMS+Escitalopram (Citoles $20 \mathrm{mg}$ tablet, Abdi Ibrahim Pharmaceuticals, Inc) (10 $\mathrm{mg} / \mathrm{kg} /$ day for 3 weeks) and CUMS+Ceftriaxone (lesef $1 \mathrm{~g}$, IM vial, Ibrahim EthemUlagayPharmaceuticals, Inc) $(200 \mathrm{mg} /$ $\mathrm{kg} /$ day for 3 weeks) ( $\mathrm{n}=10 /$ group). Bychronic unpredicted stress model, by applying various stressors to experimental animals for 40 days, depression was formed. On the 20th day of stressor application, the treatment of escitalopram and ceftriaxone were applied for two groups of experimental animals divided as randomized control, depression, escitalopram and ceftriaxone for 20 days.

\subsection{Behavioral Experiments}

\subsubsection{Chronic Unpredictable Mild Stress (CUMS) Model}

The CUMS procedure was developed by Paul Willner in the late 1980 s to model MDD more accurately, with regard to the human MDD pathogenesis (17). By combining genetic features and socio-environmental chronic stressful events, the unpredictable, chronic mild stress model can be used to study the etiological and developmental components of major depression(18). Human MDD's can only be effectively treated by chronic, but not acute, antidepressant administration (17). Furthermore, CUMS is a model to produce a depressive-like state in rodents, induced by the exposure to some natural and mild environmental stressors over a long period (4-9 weeks) that leads to the development of chronic psychological changes. Psychological changes, evoked by CUMS, are symptoms like decreased reply on rewarding stimuli including e.g. anhedonia and decreased locomotor activity (19). Anhedonia is defined as a decreased ability (the animal is not unable to experience it, but the ability is strongly reduced) to experience pleasure and is the key symptom of depression and depressive disorders. It can be assessed by measuring sucrose preference and coat state (20) of rodents.

\subsubsection{Sucrose preference test(SPT)}

Anhedonia as a core symptom of depression was evaluated by the SPT. For the adaptation period, rats were first introduced two bottles each filled with $200 \mathrm{ml} 1 \%$ sucrose solution (w/v) for 24 hours. Then, one of the bottles was taken out and replaced with an identical bottle filled with $200 \mathrm{ml}$ tap water while the other bottle was fulfilled $200 \mathrm{ml}$ of $1 \%$ sucrose solution $(\mathrm{w} / \mathrm{v})$ and kept still for a second $24 \mathrm{~h}$ period. This adaptation period was followed by $24 \mathrm{~h}$ period of food and water restriction. Following the last $24 \mathrm{~h}$ period, subjects' baseline sucrose consumptions were measured before starting CUMS procedure. This time again there were two identical bottles one of them was filled with $200 \mathrm{ml}$ of tap water and the other one was with $200 \mathrm{ml} 1 \%(\mathrm{w} / \mathrm{v})$ sucrose solution. Rats were allowed to have access to both bottles for $24 \mathrm{~h}$. At the end of $24 \mathrm{~h}$, baseline sucrose preference was calculated according to the following formula:

Sucrose preference: (consumption of sucrose solution/ consumption of total liquid) $\times 100$

Sucrose preference test was repeatedly applied 14., 21 . and 41. day (time schedule) throughout the CUMS procedure in order to evaluate anhedonia-like behavior of subjects.

\subsubsection{Locomotor activity test}

Locomotor activity was measured using an activity recording system (May Act 508 animal locomotor activity meter) consisting of a rectangular plexiglass activity cage (41 $\mathrm{x}$ $44 \times 32(\mathrm{~h})$ ) attached with 4 blocks on the side walls and an electronic unit. The blocks on the side walls ( 1 pair of opposing blocks on the opposing side walls for vertical and 
1 pair for horizontal activity) contained infra-red radiant light beams and photocells. Therefore, interruptions occurring from either vertical or horizontal movements was detected through the photocells and counted by the counter. On $40^{\text {th }}$ day (after treatment), locomotor activity was measured for $30 \mathrm{~min}$ and the surface was cleaned with $70 \%$ ethanol and left to dry before testing of each subject.

\subsubsection{Forced swim test (FST)}

The FST was used as an acute despair model to assess antidepressant like activity(16). For the habituation, rats were individually placed inside a plexiglass cylinder $(46-50 \mathrm{~cm}$ height, $20 \mathrm{~cm}$ diameter) filled with water $\left(23-25^{\circ} \mathrm{C}\right)$ to a depth of 30-31 cm and allowed to swim for $15 \mathrm{~min}$. On the testing day ( $40^{\text {th }}$ day, after treatment), 24 hours following after habituation, rats were again subjected to the same conditions except that for $5 \mathrm{~min}$ this time and each swimming sessions was video recorded. The time of immobility, the absence of any movement except the minor movements in order to keep the head above the water, was video scored by an experienced observer. The subjects were immediately removed from the water at the end of $5 \mathrm{~min}$ and gently dried by using towel in a warm atmosphere. The water was always refreshed and the temperature was checked before each subject to be tested.

\subsubsection{Elevated plus maze (EPM)}

Anxiety-like behavior of rats was evaluated by using the EPM test. The maze was a plus shaped platform located $80 \mathrm{~cm}$ above the floor consisting of 4 identical arms $(50 \mathrm{~cm}$ length $x$ $14 \mathrm{~cm}$ width); 2 opposing open and 2 opposing closed arms connected with a central open area (dimensions). The wall height of the closed arms was $50 \mathrm{~cm}$ each subject was gently placed on the central area facing one of the open arms and video recorded for a $5 \mathrm{~min}$ of duration. On $40^{\text {th }}$ day (after treatment), time latency for first entering a closed arm, time spent on open and closed arms and the number of open and closed arm entries was scored by an experienced observer. Subjects were removed from the platform at the end of $5 \mathrm{~min}$ and transferred to their home cage in the holding room. The apparatus was cleaned with $70 \%$ ethanol and allowed to dry before placing the next subject.

\subsubsection{In vivo voltammetry}

Platinum microelectrode arrays microelectrodes the realtime monitoring of rapid changes in extracellular levels of glutamate and other neuro-active molecules in the central nervous system were provided by fast analytical sensing technology (FAST). We also used S2 type (for rats), glutamate oxidase and nafioncoated multisite ceramic microelectrodes in this study. FAST and microelectrodes were obtained commercially from Pronexus Analytical AB (Stockholm, Sweden). The microelectrodes have platinum (Pt) recording sites with Pt connecting lines.
Calibration tests were performed with FAST-16 Data Acquisition Unit (Pronexus Analytical AB, Stockholm, Sweden). We used constant amperometric 0.7 voltage for in vivo voltammetry. The ceramic microelectrode amplifies head stage by being attached to a FAST 16 system. An Ag/ $\mathrm{AgCl}$ commercial electrode also attaches to the head stage and functions as the reference electrode (21).An electrode manipulator that attached to electrode on a stereotaxic frame (Stoelting Co., Wood Dale, IL, USA) was positioned above the rat brain. Our microelectrode had nafion and glutamate oxidase on the platinum surface. Glutamate oxidase converts glutamate to peroxide. On the other hand, the nafion blocks interferents. The peroxide can pass through the nafion barrier and can be detected by the platinum side of microelectrode in voltammetry system. The peroxide level is correlated with the glutamate level. The coated tip of the $\mathrm{Ag} / \mathrm{AgCl}$ reference electrode is placed into the same rat brain (22).Voltammetric analyses were after all behavioral tests have ended $\left(40^{\text {th }}\right.$ day). $\mathrm{CA} 3$ region of hippocampus in the brain was entered rat brain atlas according to coordinates $(+3.2$ on the $x$ axis, -3.6 on the $y$ axis, 3.5 on the $z$ axis) stereotaxicallyand voltammetric recordings were taken.

\subsubsection{Statistical analysis}

Statistical analysis was performed by using GraphPad Prism ${ }^{\circ}$ 5 program (GraphPad Software Inc., La Jolla, CA, USA). One-way analysis of variance (ANOVA) followed by posthoc Tukey's HSD test was used for gene expression analysis of statistics. Two-way ANOVA followed by Bonferroni test was used for analysis of SPT. Data were presented as the means $\pm S D . p<0.05$ was regarded to be statistically significant.

\section{RESULTS}

\subsection{The effect of CUMS and ceftriaxone treatment in locomotor activity.}

As shown in Table 1 rats exposed to 6-week CUMS procedure developedmarked decreases in activity compared to nonstressed control group $(p<0,01)$ in locomotor activity test. Both escitalopram and ceftriaxone treatment increased locomotor activity back to control levels compared to CUMS group significantly $(p<0,05)$ (Table 1$)$.

Table 1. The effect of CUMS and ceftriaxone treatment in locomotor activity.

\begin{tabular}{|l|l|l|}
\hline Study groups & $\mathbf{n}$ & Mean \pm SD \\
\hline Control & 10 & $1710 \pm 188$ \\
\hline CUMS & 10 & $1156 \pm 312^{* *}$ \\
\hline CUMS+Escitalopram & 10 & $1598 \pm 231^{+}$ \\
\hline CUMS+Ceftriaxone & 10 & $1515 \pm 268^{+}$ \\
\hline
\end{tabular}

All data are expressed as distance traveled $(\mathrm{cm})$ mean $\pm S D$ ( $n=10 /$ group). 


\subsection{The effect of CUMS and ceftriaxone treatments on sucrose preference in the SPT}

As shown in Figure 1, rats exposed to 6-week CUMS procedure developed anhedonia-like behavior with marked decreases in sucrose preference compared to non-stressed control group $(p<0,01)$ in SPT. Chronic administration of ceftriaxoneand escitalopram during the last 3 weeks of CUMS procedure significantly increased sucrose preference compared to CUMS group $(p<0,01)$. Our results showed that ceftriaxone treatment reversed CUMS-induced anhedonialike behaviors in rats (Figure 1).

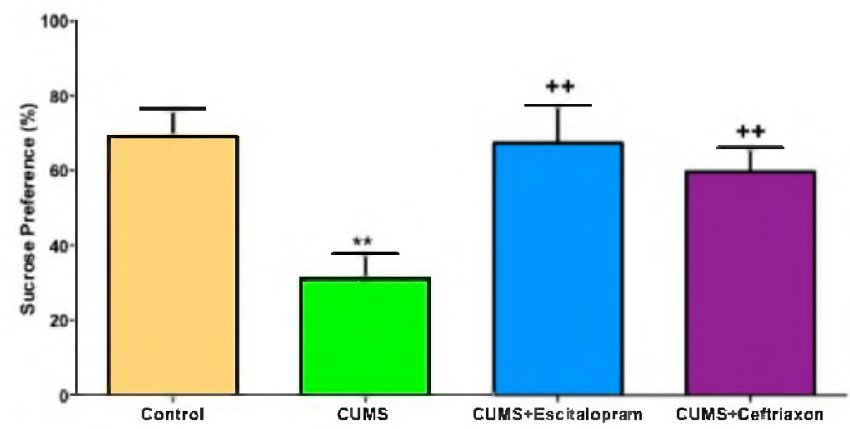

Figure 1. The effect of CUMS and ceftriaxone treatments on $\%$ sucrose preference. Data are expressed as mean \pm S.D and **: $p<0.01$ compared to control and ++: $p<0.01$ compared to CUMS group.

\subsection{The effect of CUMS and ceftriaxone treatments on immobility time in the FST}

In the FST, the time of immobility was significantly elevated in CUMS group compared to control group $(p<0,01)$. However, when CUMS-exposed rats chronically treated with ceftriaxone $(p<0,01)$ and escitalopram $(p<0,01)$, had significantly shorter duration of immobility showing that higher dose of ceftriaxone was effective in improving CUMSinduced despair-like behaviors of rats (Figure 2).

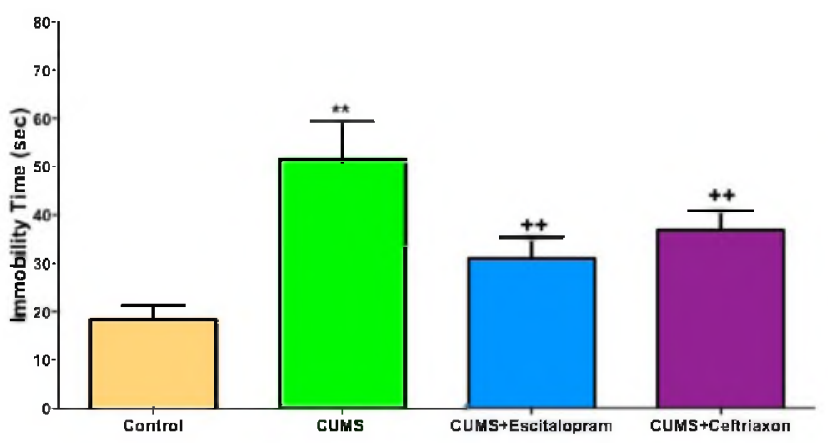

Figure 2. The effect of CUMS and ceftriaxone treatments on immobility time in the FST Data are expressed as mean $\pm S D$ and **: $p<0.01$ compared to control and ++: $p<0.01$ compared to CUMS group

\subsection{The effect of CUMS and ceftriaxone treatments the number of open arm entries in EPM test}

Number of open arms entries for 5 minutes is summarized in the table 2. The number of open-arm entries of the depression group reduced significantly when compared to the control group $(p<0.05)$. Escitalopram and ceftriaxone treatment increased significantly according to depression group $\mathrm{p}<0.05$ ).

Table 2. The effect of CUMS and ceftriaxone treatment in Evalated Plus Maze Test.

\begin{tabular}{|l|l|l|}
\hline Study groups & $\mathbf{n}$ & Mean \pm SD \\
\hline Control & 10 & $1,25 \pm 0,4629$ \\
\hline CUMS & 10 & $0,333 \pm 0,5164^{\sharp}$ \\
\hline CUMS+Escitalopram & 10 & $1,429 \pm 0,7868^{*}$ \\
\hline CUMS+Ceftriaxone & 10 & $1,167 \pm 0,4083^{*}$ \\
\hline
\end{tabular}

All data are expressed as the number of open arm entries mean $\pm S D(n=10)$ group).

\subsection{In vivo voltammetric test}

$80 \%$ of reuptaketime after peak point $\left(\mathrm{t}_{80}\right)$ in the $\mathrm{CA} 3$ region is summarized in the table 3 . Glutamate reuptake duration of the depression group significantly extended according to the control group. Compared to the depression group, escitalopram therapy markedly reduced the duration of glutamate re-uptake. But, ceftriaxone treatment did not affect the glutamate reuptake time in this region.

Table 3. Re-uptake time of glutamate on CA3 region of hippocampus.

\begin{tabular}{|l|l|l|}
\hline Study groups & $\mathbf{n}$ & MeantSD \\
\hline Control & 10 & $3 \pm 0^{*}$ \\
\hline CUMS & 10 & $4,8 \pm 0,45$ \\
\hline CUMS+Escitalopram & 10 & $3,2 \pm 0,44^{*}$ \\
\hline CUMS+Ceftriaxone & 10 & $5,17 \pm 1,94$ \\
\hline
\end{tabular}

$80 \%$ of reuptake time after peak point $\left(t_{80}\right)$ in the $C A 3$ region are summarized (sn) mean $\pm S D(n=10 /$ group).

\section{DISCUSSION}

Scientific evidences that glutamate has an effect on depression is rapidly increasing. In 2006 Mitani et al showed a positive correlation between plasma levels of glutamate and the severity of depression (23). Changes in glutamate reuptake time has been shown to play an important role in the pathogenesis of some neurological diseases. Chronic pain and ALS (amyotrophic lateral sclerosis) are just two of them. Glutamate reuptake is associated with exactly glutamate transporter activity. Drugs providing to increase the activity of glutamate transporter have been found useful in these diseases $(24,25)$. Until now functional magnetic resonance imaging, volume studies, histopathological studies and microdialysis studies were performed to reveal the relationship between depression and level of glutamate changes. But none of the mentioned studies did not show the action of glutamate in synaptic gap 
second by second.Microdialysis, which is a very important method for the measurement of neurotransmitters, and if allowed to picomolar levels of substances in a sample to be analyzed in 20 minutes is a significant disadvantage (26). Because neurotransmitters are released and taken back within seconds. In vivo voltammetry technique can detect neurotransmitter movements within one second, which can give exact information about the basal levels and the removal of glutamate parameters. We tested the levels of glutamate change by in vivo voltammetry technique in specific regions of the brain associated with depression. The monoamine hypothesis of depression can be proven to be true only by in vivo voltammetry method. Synaptic glutamate removal range shows the activity of glutamate transporters. Our results suggest that the function of glutamate transporters and glutamate movement can be determined in a selective and sensitive manner with a platinum electrode.

In our study, voltammetric analyzes were performed in the hippocampus region. The hippocampus is the mainly affected area in depression disease. The cornuammonis, one of the basic region of hippocampus, is more sensitive to glutamate toxicity. EAAT2 is one of the major glutamate transporters expressed generally in astroglial cells and is responsible for $90 \%$ of total glutamate uptake. Astrocytes compose the greater part of glial cells within the brain and may account for up to $50 \%$ of the brain's volume (27-29). In the CA3 region, ceftriaxone wasn't increased the glutamate transporter activity but escitalopram was accelerated carrier activities in this region.

We showed for the first time by this study that escitalopram accelerating the glutamate removal by voltammetry. So we say that as an antidepressant escitalopram is also effective in accelerating the reuptake of glutamate.The hippocampus is the mainly affected area in depression disease. The cornuammonis, one of the basic region of hippocampus, is more sensitive to glutamate toxicity.The CA3 region is associated exactly with depression and only escitalopram had turned to normal prolongation of reuptake period.Therefore, ceftriaxone could not accelerate the reuptake of glutamate in CA3 region (30-32).

In our study depression rat model is formed by chronic unpredicted mild stress application as described by Wilner et al. Then depression model was confirmed with behavioral tests such as locomotor activity test, elevated plus maze test and the forced swimming test (17). In the elevated plus maze test, which is a better indicator of clinical anxiety ceftriaxone treatment showed closer results to control the number of open arm entries but no closer to control of the inactivity time in the forced swimming test, which is more associated with depression. These results showed us that the ceftriaxone had more beneficial effect on anxiety rather than depression.

\section{CONCLUSION}

We showed that $80 \%$ of glutamate removal time (t80) was extended in hippocampus region in depression formed rats.
When investigating the cause of this situation, we found that the glutamate transporter activity-enhancing drugs such as ceftriaxone not show the expected improvement in the CA3 region of $\mathrm{t} 80$ period butescitalopram treatment turned back the expression levels in the same brain area. In this study it was showed that a portion of the clinical antidepressant effect of escitalopram was associated with changing the speed of glutamate reuptake and glutamate transporter expression levels.

\section{ACKNOWLEDGEMENT}

This study was supported by TUBITAK with project number 1135083. We would like to thank TUBITAK for their support in carrying out the study. A part of this study was presented at international congress by $27 \mathrm{nd}$ Europan Collage of Neuropsychopharmacology (ECNP) Berlin, Germany, 2014.

\section{REFERENCES}

[1] Skolnick P, Legutko B, Li X, Bymaster FP. Current perspectives on the development of non-biogenic amine-based antidepressants. Pharmacol Res 2001; 43(5): 411-423.

[2] Kulkarni SK, Dhir A. Current investigational drugs for major depression. Expert Opin Investig Drugs 2009; 18(6): 767-88.

[3] Lee TS, Quek SY and Krishnan KR. Molecular Imaging for Depressive Disorders. Am J Neuroradiol 2014; 35(6): 44-54.

[4] Arciniegas DB, Anderson CA, Topkoff J, McAllister TW. Mild traumatic brain injury: a neuropsychiatric approach to diagnosis, evaluation, and treatment. Neuropsychiatr Dis Treat 2005; 1(4): 311-327.

[5] Duman RS. Pathophysiology of depression and innovative treatments: remodelling glutamatergic synaptic connections. Dialogues Clin Neuroscie 2014; 16: 11-27.

[6] Zhu X, Ye G, Wang Z, Luo J, Hao X. Sub-anesthetic doses of ketamine exert antidepressant-like effects and upregulate the expression of glutamate transporters in the hippocampus of rats. Neurosci Lett 2017; 639(3): 132-137.

[7] Czéh B, Fuchs E, Wiborg O, Simong M. Animal models of major depression and their clinical implications. Prog Neuropsychopharmacol Biol Psychiatry 2016; 64: 293-310.

[8] Willner P. Validity, reliability and utility of the chronic mild stress model of depression: a 10-year review and evaluation. Psychopharmacology 1997; 134(4): 319-329.

[9] Willner P. Chronic Mild Stress (CMS) Revisited: Consistency and Behavioural-Neurobiological Concordance in the Effects of CMS, Neuropsychobiology 2005; 52: 90-110.

[10] Willner P. Chronic mild stress-induced anhedonia: A realistic animal model of depression. Neurosci Biobehav Rev 1992; 16 (4): 525-534.

[11] Helena M. Abelaira HM, Reus GZ, Quevedo J. Animal models as tools to study the pathophysiology of depression. Rev Bras Psiquiatr 2013; 35(2): 112-120.

[12] Deussing JM. Animal models of depression, Drug Discov Today 2006; 3(4): 375-383.

[13] Wiborg 0 . Chronic mild stress for modeling anhedonia. Cell Tissue Res 2013; 354(1): 155-169.

[14] Haapakoski R, Ebmeier KP, AleniuscH,Kivimäki M. Innate and adaptive immunity in the development of depression: An 
update on current knowledge and technological advances. Prog Neuropsychopharmacol Biol Psychiatry 2016; 66: 63-72.

[15] Kaufmann FN, Costa AP, Ghisleni G, Diaz AP, Rodrigues ALS Peluffo $H$, Kaster MP. NLRP3 inflammasome-driven pathways in depression: Clinical and preclinical findings. Brain Behav Immun. 2017; 64: 367-383.

[16] Valtcheva S, Venance L. Astrocytes gate Hebbian synaptic plasticity in the striatum. Nat Commun 2016; 20(7): 1-17.

[17] Willner P, Towell A, Sampson D, Sophokleous S, Muscat R. Reduction of sucrose preference by chronic unpredictable mild stress, and its restoration by a tricyclic antidepressant. Psychopharmacol 1987; 93(3): 358-364.

[18] Yazir Y, Utkan T, Aricioglu F. Inhibition of neuronal nitric oxide synthase and soluble guanylate cyclase prevents depressionlike behaviour in rats exposed to chronic unpredictable mild stress. Basic Clin Pharmacol Toxicol 2012; 111(3): 154-60.

[19] Jiang P, Zhang WY, Li HD, Cai HL, Liu YP, Chen LY. Stress and vitamin D: altered vitamin D metabolism in both the hippocampus and myocardium of chronic unpredictable mild stress exposed rats. Psychoneuroendocrinology 2013; 38(10): 2091-2098.

[20] Nollet M, Le Guisquet AM, Belzung C. Models of depression: unpredictable chronic mild stress in mice. Curr Protoc Pharmacol. 2013; 5 : 5.

[21] Burmeister J, Kota C, Maughan RL, Spokas JJ, Coderre JA, Ma $\mathrm{R}$, Wielopolski L. A conducting plastic simulating brain tissue. Med Phys 2000; 27(11): 2560-2564.

[22] Hacimuftuoglu A, Tatar A, Cetin D, Taspinar N, Saruhan F, Okkay $\mathrm{U}$, Turkez H, Unal D, Stephens RL Jr, Suleyman H. Astrocyte/ neuron ratio and its importance on glutamate toxicity: an in vitro voltammetric study. Cytotechnology 2016; 68(4): 14251433.

[23] Mitani H, Shirayama Y, Yamada T, Maeda K, Ashby CR, Kawahara R. Correlation between plasma levels of glutamate, alanine and serine with severity of depression. Prog Neuropsychopharmacol Biol Psychiatry 2006; 30: 1155-1158.
[24] Rothstein JD, Kammen VM, Levey Al, Martin LJ, Kuncl RW. Selective loss of glial glutamate transporter GLT-1 in amyotrophic lateral sclerosis. Ann Neurol 1995; 38: 73-84.

[25] Stephens RL. Glutamate transporter activators as antinociceptive agents. Eurasian J Med 2011; 43: 182-185.

[26] Jaquins-Gerstl A, Michael AC. Comparison of the brain penetration injury associated withmicrodialysis and voltammetry. J Neurosci Methods 2009; 183(2): 127-135.

[27] Medina A, Burke S, Thompson RC, Bunney W, Myers RM, Schatzberg A, Akil H, Watson SJ. Glutamate transporters: a key piece in the glutamate puzzle of major depressive disorder. J Psychiatr Res 2013; 47: 1150-1156.

[28] Kim K, Seok-Guen L, KegelmanTP, Su ZZ, Das SK, Dash R, Dasgupta S, Barral PM, Hedvat M, Diaz P, Reed JC, Stebbins JL, Pellecchia M, Sarkar D, Fisher PB. Role of excitatory amino acid transporter-2 (EAAT2) and glutamate in neurodegeneration: opportunities for developing novel therapeutics. J Cell Physiol 2011; 226(10): 2484-93.

[29] Tower DB, Young OM. The activities of butyrylcholinesterase and carbonic anhydrase, the rate of anaerobic glycolysis, and the question of a constant density of glial cells in cerebral cortices of various mammalian species from mouse to whale. J Neurochem 1973; 20(2): 269-78.

[30] Medina A, Burke S, Thompson RC, Bunney W, Myers RM, Schatzberg A, Akil H, Watson SJ. Glutamate transporters: a key piece in the glutamate puzzle of major depressive disorder. J Psychiatr Res 2013; 47: 1150-1156.

[31] Tordera RM, Garcia-Garcia AL, Elizalde N, Segura V, Aso E, Venzala E, Ramirez MJ, Del Rio J. Chronic stress and impaired glutamate function elicit a depressive-like phenotype and common changes in gene expression in the mouse frontal cortex. Eur Neuropsychopharmacol 2011; 21: 23-32.

[32] Yang H, Spence JS, Devous MD, Briggs RW, Goyal A, Xiao H, Yadav H, Adinoff B. Striatal-limbic activation is associated with intensity of anticipatory anxiety. Psychiatry Res 2012; 204: 123-131.

How to cite this article: Binnetoglu D, Aricioglu F, Ozcan H, Okkay U, Hacimuftuoglu A. Antidepressant-like effects of ceftriaxone in chronic unpredictable mild stress model in rats: The importance of reuptake time of glutamate. Clin Exp Health Sci 2019; 9: $294-299$. DOI: $10.33808 /$ clinexphealthsci.613963 